\title{
Emergence of divergent enterovirus (EV) D68 sub-clade D1 strains, northern Italy, September to October 2018
}

Laura Pellegrinelli ${ }^{1}$, Federica Giardina ${ }^{2}$, Giovanna Lunghi ${ }^{3}$, Sara Colonia Uceda Renteria ${ }^{3}$, Letizia Greco ${ }^{3}$, Alice Fratini ${ }^{2}$, Cristina Galli $^{1}$, Antonio Piralla ${ }^{2}$, Sandro Binda ${ }^{1}$, Elena Pariani ${ }^{1}$, Fausto Baldanti ${ }^{2,4}$

1. Department of Biomedical Sciences for Health, University of Milan, Milan, Italy

2. Molecular Virology Unit, Microbiology and Virology Department, Fondazione IRCCS Policlinico San Matteo, Pavia, Italy

3. Microbiology and Virology Unit, Fondazione IRCCS Ca' Granda Ospedale Maggiore Policlinico, Milan, Italy

4. Department of Clinical, Surgical, Diagnostic and Pediatric Sciences, University of Pavia, Pavia, Italy

Correspondence: Antonio Piralla (a.piralla@smatteo.pv.it)

Citation style for this article:

Pellegrinelli Laura, Giardina Federica, Lunghi Giovanna, Uceda Renteria Sara Colonia, Greco Letizia, Fratini Alice, Galli Cristina, Piralla Antonio, Binda Sandro, Pariani Elena, Baldanti Fausto. Emergence of divergent enterovirus (EV) D68 sub-clade D1 strains, northern Italy, September to October 2018 . Euro Surveill. 2019;24(7):pii=1900090. https://doi.org/10.2807/1560-7917.ES.2018.24.7.1900090

Article submitted on 31 Jan 2019 / accepted on 13 Feb 2019 / published on 14 Feb 2019

Between September and October 2018, an enterovirus D68 (EV-D68) outbreak occurred in patients hospitalised with severe acute respiratory infection in northern Italy; 21 laboratory-confirmed cases were reported. Phylogenetic analysis revealed that $16 / 20$ of the EV-D68 sequences belonged to a divergent group within the sub-clade D1. Since its upsurge, EV-D68 has undergone rapid evolution with the emergence of new viral variants, emphasising the need for molecular surveillance that include outpatients with respiratory illness.

An enterovirus D68 (EV-D68) outbreak was recognised at a major tertiary centre and research hospital in Milan and Pavia in the Lombardy region, northern Italy, respectively, between September and October 2018. Here, we present the molecular and clinical characteristics of the confirmed EV-D68 cases detected during the outbreak sustained by EV-D68 sub-clade D1 strains.

\section{Enterovirus D68 detection}

From 1 August to 30 October 2018, routine laboratory testing on 853 respiratory samples, collected from patients hospitalised with respiratory infection, were analysed using molecular assays to detect causing respiratory viruses and bacteria. Of these, 91 (10.7\%) tested positive for rhinovirus/EV and were further analysed using a real-time one-step RT (reversetranscription)-PCR assay targeting the 5' untranslated region (5'UTR) of EV-D68 [1,2]; 21 samples were positive for EV-D68 and no other viruses or bacteria were identified. Among EV-D68-positive samples, 10 were nasopharyngeal aspirates (NPA), eight nasopharyngeal swabs (NS) and three broncho-alveolar lavages (BAL).

\section{Characteristics of patients with enterovirus D68 infection}

Of 21 EV-D68-positive cases, the median age was 18 years (range: 1 month-84 years) and 14 were male. An underlying or concurrent condition was reported for 10 patients (Table). Underlying conditions were: genetic/metabolic disorder ( $n=4)$; leukaemia ( $=3$ ); cystic fibrosis $(n=1)$; chronic heart disease ( $=1)$; and lymphoma $(n=1)$. Sixteen of 21 EV-D68positive patients had signs and/or symptoms of lower respiratory tract infection (LRTI) and five showed symptoms of mild respiratory infection (Table). Among LRTI cases, five patients required intensive care unit (ICU) admission due to respiratory failure.

\section{Phylogenetic and molecular analysis}

Complete viral protein (VP) 1 nt sequences [3] were obtained for 20 of 21 EV-D68 strains (GenBank accession numbers: MK301336-MK301355). A maximum likelihood phylogenetic tree was inferred using IQ-Tree web server (v1.6.8) [4] and the robustness of branches was evaluated using the Shimodaira-Hasegawa approximate likelihood-ratio test (SH-aLRT) and ultrafast bootstrap approximation tests.

Unexpectedly, most (16/20) sequences clustered into a distinct genetic group within the $\mathrm{D}$ clade, with a bootstrap value of $99 \%$ (Figure). Clade D has recently been reclassified into two sub-clades (referred as D1 and D2) [5,6]. The Italian strains clustered with one Taiwanese strain collected in 2016, one United States (US) strain collected in 2017 and 13 French strains collected in 2018 (shown in green in Figure), which together composed a divergent D1-like group. The average nt identity between EV-D68 strains belonging to this divergent group was $98.0 \%$; the sequences shared an average nt identity of 96.3\% (range: $94.7-$ $97.8 \%)$ with all available $D_{1}$ strains $(n=69)$ retrieved 
Characteristics of patients with enterovirus D68 infection, northern Italy, 1 August-30 October $2018(\mathrm{n}=21)$

\begin{tabular}{|c|c|c|c|c|c|c|c|}
\hline $\begin{array}{l}\text { Age group } \\
\text { (years) }\end{array}$ & $\begin{array}{l}\text { Underlying } \\
\text { disease }\end{array}$ & Respiratory illness & $\begin{array}{c}\text { ICU } \\
\text { admission }\end{array}$ & $\begin{array}{c}\text { Type of } \\
\text { sample } \\
\text { analysed }\end{array}$ & $\begin{array}{l}\text { Sample } \\
\text { collection } \\
\text { month }\end{array}$ & Strain name & $\begin{array}{l}\text { EV-D68 } \\
\text { sub-clade }\end{array}$ \\
\hline $80-89$ & Yes & Bronchiolitis & No & NS & September & EV-ITA-684-2018 & D1-like \\
\hline $20-29$ & Yes & $\begin{array}{c}\text { SARI and bronchial } \\
\text { asthma }\end{array}$ & Yes & NPA & September & EV-ITA-685-2018 & D1-like \\
\hline $40-49$ & Yes & Bronchiolitis & No & NS & September & EV-ITA-686-2018 & D1-like \\
\hline $60-69$ & NA & Rhinorrhea & No & NS & September & EV-ITA-19478-2018 & D1-like \\
\hline $1-9$ & No & Pneumonia & No & NS & September & EV-ITA-19704-2018 & D1-like \\
\hline $70-79$ & NA & Pneumonia & No & BAL & September & EV-ITA-19705-2018 & D1-like \\
\hline$\ll 1$ & No & $\begin{array}{c}\text { Recurrent } \\
\text { bronchospasms }\end{array}$ & No & NS & September & EV-ITA-19957-2018 & D1-like \\
\hline $70-79$ & Yes & Pneumonia & No & NS & September & EV-ITA-689-2018 & D1-like \\
\hline$\ll 1$ & No & Febrile wheezing & No & NPA & September & EV-ITA-20100-2018 & D1-like \\
\hline $30-39$ & NA & Rhinorrhea and cough & No & NS & September & EV-ITA-20181-2018 & D1-like \\
\hline $10-19$ & Yes & $\begin{array}{c}\text { SARI and bronchial } \\
\text { asthma }\end{array}$ & Yes & BAL & September & EV-ITA-687-2018 & D1-like \\
\hline $1-9$ & No & $\begin{array}{l}\text { SARI and bronchial } \\
\text { asthma }\end{array}$ & Yes & NPA & September & EV-ITA-688-2018 & B2-like \\
\hline $20-29$ & Yes & $\begin{array}{l}\text { SARI and bronchial } \\
\text { asthma }\end{array}$ & No & NPA & September & EV-ITA-690-2018 & D1-like \\
\hline $10-19$ & No & $\begin{array}{c}\text { SARI and bronchial } \\
\text { asthma }\end{array}$ & Yes & NPA & September & EV-ITA-683-2018 & D1-like \\
\hline $1-9$ & Yes & Rhinorrhea and cough & No & NPA & September & EV-ITA-693-2018 & D1-like \\
\hline $1-9$ & Yes & $\begin{array}{c}\text { SARI and bronchial } \\
\text { asthma }\end{array}$ & Yes & NPA & September & EV-ITA-695-2018 & B2-like \\
\hline $60-69$ & Yes & Pneumonia & No & NS & October & EV-ITA-694-2018 & D1-like \\
\hline $1-9$ & No & $\begin{array}{c}\text { SARI and bronchial } \\
\text { asthma }\end{array}$ & No & NPA & October & EV-ITA-696-2018 & NA \\
\hline $1-9$ & No & Rhinorrhea and cough & No & NPA & October & EV-ITA-751-2018 & B2-like \\
\hline$\ll 1$ & No & $\begin{array}{c}\text { SARI and bronchial } \\
\text { asthma }\end{array}$ & No & NPA & October & EV-ITA-753-2018 & B2-like \\
\hline $80-89$ & Yes & Cough and pneumonia & No & BAL & October & EV-ITA-757-2018 & D1-like \\
\hline
\end{tabular}

BAL: broncho-alveolar lavage; EV-D68: enterovirus D68; ICU: intensive care unit; NA: not available; NPA: nasopharyngeal aspirate; NS: nasal swab; SARI: severe acute respiratory infection.

from GenBank and 93.2\% (range: 92.1-94.4\%) with all available D2 strains $(n=7)$. The remaining four EV-D68 strains detected during the outbreak belonged to subclade B2 and clustered with strains collected in 2018 in France.

All VP1 sequences of EV-D68 strains belonging to the divergent D1-like group differed from sub-clade $D_{1}$ by the $\mathrm{V}_{2} 99 \mathrm{l}$ change. A series of polymorphisms, such as T98V and $1243 \mathrm{~V}$, was observed in several subgroups of sequences. In addition, 12 of 16 Italian sequences harboured the I17M change and the remaining four strains showed the $13 \mathrm{~V}$ change. Four polymorphisms ( $98 \mathrm{~V}, 102 \mathrm{~L}, 142 \mathrm{~T}$ and $143 \mathrm{D}$ ) observed in the Italian strains were within codons of protein motifs corresponding to the $\mathrm{BC}$ and DE loops (Figure).

\section{Discussion and conclusion}

In 2008, EV-D68 was first reported in Italy $[3,7]$. In 2014 and 2016, the US and several European countries experienced a surge in the number of severe acute respiratory infection cases caused by EV-D68 infection, sustained by strains belonging to multiple EV-D68 clades $[8,9]$;

These outbreaks, in particular the one that occurred in 2016, were chronologically and geographically superimposed by an unprecedented cluster of acute flaccid myelitis (AFM) cases, with a striking clinical similarity to poliomyelitis in patients with respiratory prodromal illness [8]. Two AFM cases associated with EV-D68 were recognised in the same period in Italy $[10,11]$. The association between EV-D68 infection and AFM was confirmed recently [12-14], triggering public health concern [15]. Following the 2014 outbreak, improved EV-D68 surveillance has revealed EV-D68 


\section{FIGURE}

Phylogenetic rooted tree inferred by maximum likelihood of complete enterovirus D68 VP1 sequences, northern Italy, 1 August-30 October $2018(\mathrm{n}=20)$

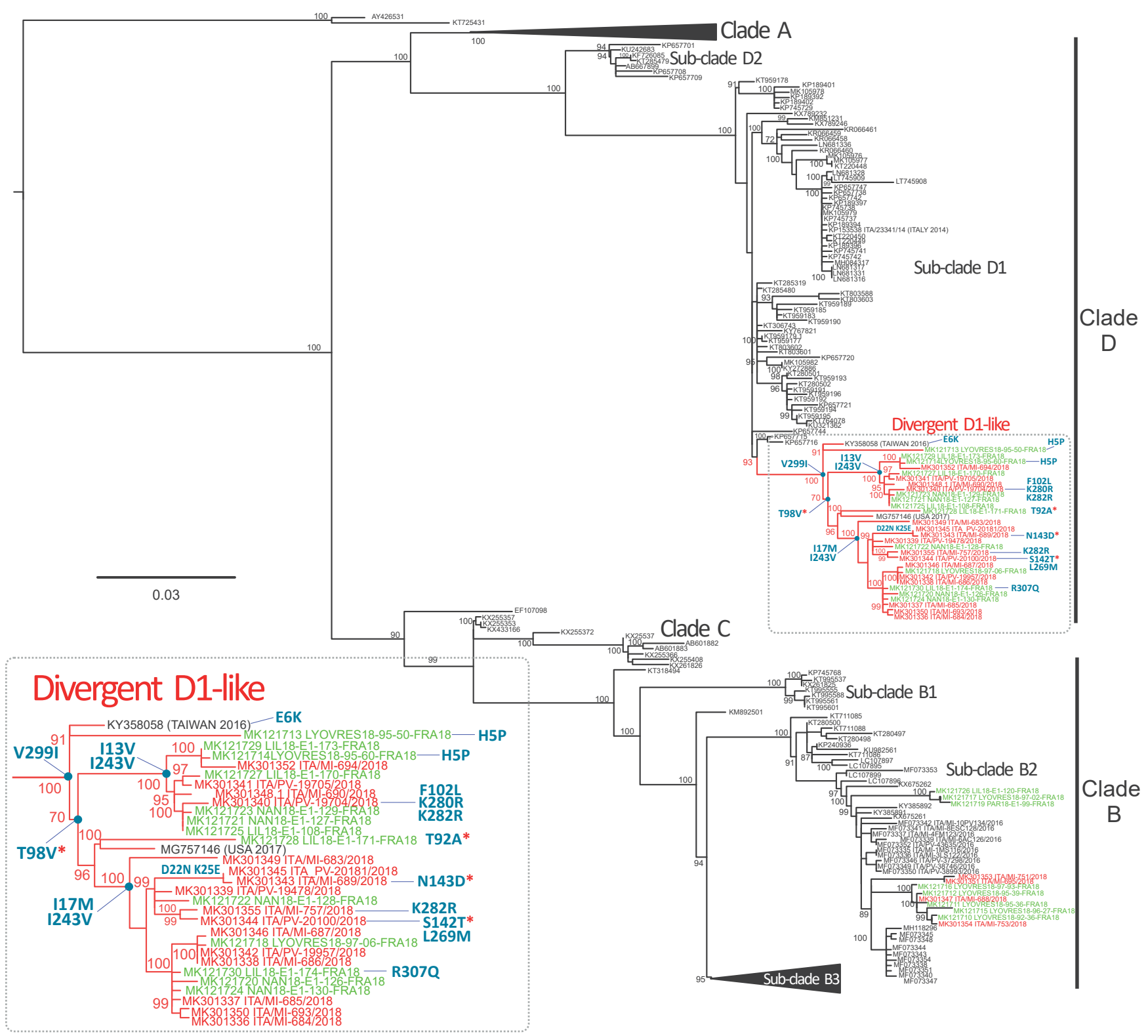

EV-D68: enterovirus D68; VP: viral protein.

A Tamura 3-parameter substitution model with a gamma distribution among-site rate variation was selected as the best-fit model by ModelTest in MEGA software 7.0.21 [21]. Significant branch support values $(>70 \%)$ are given for maximum-likelihood implementations (IQTREE bootstrap support) and are reported near the branch nodes. The EV-D68 strains identified in this study are reported in red. EV-D68 strains identified in France in 2018 [22] are reported in green. Amino acids residues characterising divergent D1-like strains are indicated in blue, including mutations in the $B C$ and $D E$ loops reported with an asterisk.

circulation in several countries in Europe and North America $[2,8,16]$.

In our study, none of EV-D68-positive patients showed signs or symptoms of neurological impairment. However, in line with previous reports [17-19], 16 of 21 EV-D68-positive patients in our series had severe respiratory infections and five of them required ICU admission. A limitation of our series is that we included only inpatients with clinical illness that required hospitalisation, thus the severity of EV-D68 infections may have been overestimated. In addition, presence of underlying conditions in some patients could have made the outcome of EV-D68 infection more severe. Involving general practitioners in EV-D68 surveillance among outpatients with respiratory illness could help improve the current knowledge regarding the clinical characteristics of EV-D68 infection and the proportion of severe disease among those infected. 
The phylogenetic analysis of EV-D68 sequences indicates that, over the past two decades, four clades (A to D) and multiple sub-lineages have emerged and spread rapidly worldwide $[5,8,16]$. The phylogenetic analysis of EV-D68 strains identified during the outbreak in Italy and in France (August-November 2018), underlined a wave of EV-D68 activity in Europe and revealed the presence of EV-D68 strains segregating into a divergent group within sub-clade D1 sequences. Based on the genetic distance analyses, this group of new D1-like sequences showed less genetic divergence from D1 (average 3.7\%) than that previously observed between $D_{1}$ and $D_{2}$ (average $5.2 \%$ ) [6]; we thus cannot consider this group as a new sub-clade. The re-emergence of EV-D68 strains belonging to two 'old' sub-clades (B2 and D1) suggests that amino acid variations have occurred over the last few years. Several amino acidic changes were observed in residues located on the surface $B C$ and DE loops resulting in a diversification of antigenic epitopes [20]. These polymorphisms may be signatures of these divergent $D_{1}$ strains, it is unclear, however, whether they possess a selective advantage. Overall, a continuous emergence and replacement of clades and sub-clades with a biennial pattern of circulation has been observed worldwide $[5,8]$.

Our findings raise attention of the rapid emergence of new EV-D68 variants that could be associated with unexpected and possible severe clinical syndromes such as AFM. So far there is no direct evidence of the association of neurological impairment with a specific EV-D68 variant. Studies are needed to investigate the host-pathogen relationship during infection and to clarify the association between genetic diversity and clinical outcome. Sharing virological, epidemiological and clinical data on EV-D68 will enable a better understanding of the elusive pathogenesis of EV-D68.

\section{Acknowledgements}

This work was supported by the Ministero della Salute (Fondazione IRCCS Policlinico San Matteo, Ricerca Corrente grant 80622).

\section{Conflict of interest}

None declared.

\section{Authors' contributions}

Conceived and designed the study: AP, EP, SB, and FB. Samples collection: FG, AF, GL, and SCUR. Performed experiments: LP, FG, AF, SCUR, LG and CG. Data processing and analysis: LP, FG, AP and EP. Drafted the manuscript: LP, AP, and EP. All authors read and approved the final manuscript. All authors fulfilled the ICMJE authorship criteria.
References

1. Piralla A, Girello A, Premoli M, Baldanti F. A new real-time reverse transcription-PCR assay for detection of human enterovirus 68 in respiratory samples. J Clin Microbiol. 2015;53(5):1725-6. https://doi.org/10.1128/JCM.03691-14 PMID: 25694533

2. Poelman R, Schuffenecker I, Van Leer-Buter C, Josset L, Niesters HGM, Lina B. European surveillance for enterovirus D68 during the emerging North-American outbreak in 2014. J Clin Virol. 2015;71:1-9. https://doi.org/10.1016/j. jcv.2015.07.296 PMID: 26364237

3. Piralla A, Girello A, Grignani M, Gozalo-Margüello M, Marchi A, Marseglia G, et al. Phylogenetic characterization of enterovirus 68 strains in patients with respiratory syndromes in Italy. J Med Virol. 2014;86(9):1590-3. https://doi.org/10.1002/ jmv.23821 PMID: 24155220

4. Trifinopoulos J, Nguyen LT, von Haeseler A, Minh BQ. W-IQTREE: a fast online phylogenetic tool for maximum likelihood analysis. Nucleic Acids Res. 2016;44(W1):W232-5. https://doi. org/10.1093/nar/gkw256 PMID: 27084950

5. Gong YN, Yang SL, Shih SR, Huang YC, Chang PY, Huang CG, et al. Molecular evolution and the global reemergence of enterovirus D68 by genome-wide analysis. Medicine (Baltimore). 2016;95(31):e4416. https://doi.org/10.1097/ MD.0000000000004416 PMID: 27495059

6. Yip CCY, Lo JYC, Sridhar S, Lung DC, Luk S, Chan KH, et al. First Report of a Fatal Case Associated with EV-D68 Infection in Hong Kong and Emergence of an Interclade Recombinant in China Revealed by Genome Analysis. Int J Mol Sci. 2017;18(5):E1065. PMID: 28509856

7. Piralla A, Lilleri D, Sarasini A, Marchi A, Zecca M, Stronati M, et al. Human rhinovirus and human respiratory enterovirus (EV68 and EV104) infections in hospitalized patients in Italy, 20082009. Diagn Microbiol Infect Dis. 2012;73(2):162-7. https://doi. org/10.1016/j.diagmicrobio.2012.02.019 PMID: 22494556

8. Holm-Hansen CC, Midgley SE, Fischer TK. Global emergence of enterovirus D68: a systematic review. Lancet Infect Dis. 2016;16(5):e64-75. https://doi.org/10.1016/S14733099(15)00543-5 PMID: 26929196

9. Piralla A, Principi N, Ruggiero L, Girello A, Giardina F, De Sando E, et al. Enterovirus-D68 (EV-D68) in pediatric patients with respiratory infection: The circulation of a new $\mathrm{B}_{3}$ clade in Italy. J Clin Virol. 2018;99-100:91-6. https://doi.org/10.1016/j. jcv.2018.01.005 PMID: 29396353

10. Giombini E, Rueca M, Barberi W, Iori AP, Castilletti $C$ Scognamiglio P, et al. Enterovirus D68-Associated Acute Flaccid Myelitis in Immunocompromised Woman, Italy. Emerg Infect Dis. 2017;23(10):1690-3. https://doi.org/10.3201/ eid2310.170792 PMID: 28930024

11. Esposito S, Chidini G, Cinnante C, Napolitano L, Giannini A, Terranova L, et al. Acute flaccid myelitis associated with enterovirus-D68 infection in an otherwise healthy child. Virol J. 2017;14(1):4. https://doi.org/10.1186/s12985-016-0678-0 PMID: 28081720

12. Messacar K, Asturias EJ, Hixon AM, Van Leer-Buter C, Niesters HGM, Tyler KL, et al. Enterovirus D68 and acute flaccid myelitis-evaluating the evidence for causality. Lancet Infect Dis. 2018;18(8):e239-47. https://doi.org/10.1016/S14733099(18)30094-X PMID: 29482893

13. Hixon AM, Yu G, Leser JS, Yagi S, Clarke P, Chiu CY, et al. A mouse model of paralytic myelitis caused by enterovirus D68. PLoS Pathog. 2017;13(2):e1006199. https://doi.org/10.1371/ journal.ppat.1006199 PMID: 28231269

14. Dyda A, Stelzer-Braid S, Adam D, Chughtai AA, Maclntyre CR. The association between acute flaccid myelitis (AFM) and Enterovirus D68 (EV-D68) - what is the evidence for causation? Euro Surveill. 2018;23(3):17-00310. https:// doi.org/10.2807/1560-7917.ES.2018.23.3.17-00310 PMID: 29386095

15. Devi S. US CDC task force to investigate acute flaccid myelitis cases. Lancet. 2018;392(10162):2339. https://doi.org/10.1016/ S0140-6736(18)33012-5 PMID: 30527604

16. Eshaghi A, Duvvuri VR, Isabel S, Banh P, Li A, Peci A, et al. Global Distribution and Evolutionary History of Enterovirus D68, with Emphasis on the 2014 Outbreak in Ontario, Canada. Front Microbiol. 2017;8:257. https://doi.org/10.3389/ fmicb.2017.00257 PMID: 28298902

17. Cottrell S, Moore C, Perry M, Hilvers E, Williams C, Shankar AG. Prospective enterovirus D68 (EV-D68) surveillance from September 2015 to November 2018 indicates a current wave of activity in Wales. Euro Surveill. 2018;23(46):1800578. https:// doi.org/10.2807/1560-7917.ES.2018.23.46.1800578 PMID: 30458915

18. Midgley CM, Watson JT, Nix WA, Curns AT, Rogers SL, Brown $B A$, et al. Severe respiratory illness associated with a 
nationwide outbreak of enterovirus D68 in the USA (2014): a descriptive epidemiological investigation. Lancet Respir Med. 2015;3(11):879-87. https://doi.org/10.1016/S22132600(15)00335-5 PMID: 26482320

19. Martin G, Li R, Cook VE, Carwana M, Tilley P, Sauve L, et al. Respiratory Presentation of Pediatric Patients in the 2014 Enterovirus D68 Outbreak. Can Respir J. 2016;2016:8302179. https://doi.org/10.1155/2016/8302179 PMID: 27610028

20. Linsuwanon P, Puenpa J, Suwannakarn K, Auksornkitti V, Vichiwattana P, Korkong S, et al. Molecular epidemiology and evolution of human enterovirus serotype 68 in Thailand, 20062011. PLoS One. 2012;7(5):e35190. https://doi.org/10.1371/ journal.pone.0035190 PMID: 22586446

21. Kumar S, Stecher G, Tamura K. MEGA7: Molecular Evolutionary Genetics Analysis version 7.0 for bigger datasets. Mol Biol Evol. 2016;33(7):1870-4. https://doi.org/10.1093/molbev/ msw054 PMID: 27004904

22. Bal A, Sabatier M, Wirth T, Coste-Burel M, Lazrek M, Stefic K, et al. Emergence of enterovirus D68 clade D1, France, August to November 2018. Euro Surveill. 2019;24(3):1800699. https:// doi.org/10.2807/1560-7917.ES.2019.24.3.1800699 PMID: 30670143

\section{License and copyright}

This is an open-access article distributed under the terms of the Creative Commons Attribution (CC BY 4.0) Licence. You may share and adapt the material, but must give appropriate credit to the source, provide a link to the licence, and indicate if changes were made.

This article is copyright of the authors or their affiliated institutions, 2019. 